\title{
LA ENSEÑANZA DE UNA LENGUA EXTRANJERA CARA A LA REFORMA EDUCATIVA
}

\section{Carmen Castro Castro}

\begin{abstract}
This paper is a survey on the state of Foreign language teaching and learning in Primary and Secondary Spanish schools. The General Educative Reform (L.O.G.S.E.) is faced by foreign language teachers at this moment and that implies a larger amount of requirements to become a L2 teacher: higher level of performance and the assumption of a communicative and interactive methodology. In spite of general agreement about positive effects on Primary L2 learning, it is noticeable the absence of teachers'framework as a variable factor to be taken into account at the same stage as the rest of the factors, students, methods and context. Quantitative and qualitative data elicited from different kinds of questionnaires are brought forward in this paper showing, theoretically, a level of adecuacy to the new methodological approaches. However contradictions are often found among teachers inquiry answers. That leads us to demonstrate that teachers are not aware of their own performances in classroom settings and claim the necessity for second language classroom research. Two main fields are proposed as a further research in L2. The first one is based on the assumption that teachers'work requires to make decisions in complex environments and what they actually do in class is guided by their thoughts, judments and decisions which in turn will be influenced by their training and personal characteristics. The second proposal is the choice of a research method for L2 teachers in such a way that they become aware of their planning and practical action in classroom settings.
\end{abstract}

La enseñanza del Segundo Idioma en Primaria y en Secundaria es motivo de numerosas publicaciones en las que se manifiestan sus carencias, problemas y necesidades. Generalmente se describe la metodología empleada, en el contexto escolar real de los Centros que están incorporando, a trancas y barrancas, la Reforma. No tan públicamente, sino en Seminarios Permanentes o reuniones profesionales, se habla también de la escasa formación del actual profesorado que imparte el Segundo Idioma y de las mínimas facilidades que se le dan a éste para su adaptación a las exigencias de la L.O.G.S.E. Todo ello influye sin duda alguna en la enseñanza; pero, para saber cómo se desarrolla con detalle la enseñanza de la L2, deberíamos preguntarnos: ¿Qué ocurre dentro del aula de L2?

En la enseñanza en general, y en la de una L2 en particular, la libertad de cátedra o el papel externo del Inspector convierten el interior de las aulas en algo muy personal y no sujeto a revisión profunda alguna, puesto que aún no es muy usual la investigación educativa salvo en contadas ocasiones y, cuando ésta existe, se queda a nivel teórico.

It is curious how rarely we collect data on different aspects of the teacher's classroom performance. In the field of sports, for example, the athlete watches closely the statistical data that summarize observations of his performace- (...) Also, athlete are exposed constantly to videotape replays of their performances so that they can perfect their techniques. In professions such as medicine, business, and law, the practitioner has access to many 
indicators that directly reflect quality of performance. (...) Additionally, practitioners often hear expression of satisfaction or dissatisfaction from their clients. We need to provide teachers whith similar indicators of performance, based on direct or indirect observation. (Acheson, Gall 1992:10)

La investigación educativa es, no obstante, una corriente con gran número de aportaciones dentro de la investigación en educación, existiendo abundantes estudios con este mismo enfoque sobre la L2 realizados en el extranjero; aunque no están exentos aún de falta de consideración por parte de los profesores que la estiman demasiado lejana a sus preocupaciones diarias.

The gulf between research and the teaching profession has ensured that many programmes are not related to professional concerns and interest of teachers and students. Priorities for research too often reflect the interest of academic researchers or central office administrators not school people. Teachers and student in the classrooms are rarely actively engaged in the research. Within the experimental framework the researcher protects his or her independence for the sake of "objectivity". The tacit knowledge of teachers is devalued. (...) Not surprisingly we the practising teachers have come to distrust and reject theoretical research and the researcher who takes but does not give. (Beasley, Riordan 1981:60)

Refiriéndonos a la enseñanza de la L2 en Andalucía, en una situación de cambio producida por la incorporación de la Ley de Reforma Educativa a los centros de enseñanza Primaria y Secundaria, podemos comprobar que ha habido una importante modificación, a nivel teórico, en el enfoque de la enseñanza de la L2; si antes se trataba de aprender la L2, ahora, interpretando el D.C.B., se intenta comunicar en la L2, enfoque lingüístico de tipo funcional comunicativo al que hay que añadirle su incardinación en unos principios de psicología de la instrucción basados en el cognitivismo y contructivismo.

El nuevo ámbito didáctico y de investigación del D.C.B, sin embargo, llega a las aulas en forma de libros de texto acompañados de soporte audiovisual y esta concreción hace desaparecer, en la mayoría de los casos, la amplitud y libertad del enfoque volviendo a constituir "otra metodología más" que los profesores deberían poner en práctica.

The growing interest in classroom-oriented research, and indications by teachers that they would like to carry out their own research, are signs of maturity withing the language teaching profession. They mark a departure from the "follow the right method'approach, with its implication that somewhere there is a correct method waiting to be discovered which will work for all learners in all situations and circumstances. (...) Rather than adopting new methods, materials of ideas and judging their efficacy on intuitive grounds, it is far more satisfactory, and professionally rewarding, to establish a small-scale classroom experiment to monitor, observe and document the effect of the new methods or materials on learner language, learning outcomes, classroom climate and patterns of group interaction. (...) In addition this alternative orientation seeks to derive principles for teaching from the close observation and documentation of what actually happens in the classroom rather than uncritically importing and applying ideas from outside. (Nunam 1989: 97-98)

Desde nuestro punto de vista, el D.C.B. en sí, y aún más sus concreciones en materiales didácticos, olvidan la figura del profesor de L2, olvidan, al parecer, que éste también pertenece a un contexto social y cultural, que ha tenido una formación determinada y quizás un cierto "estilo de enseñanza"; Por el hecho de ser profesor no es ya, de forma definitiva e invariable, un ente capaz de desarrollar a la perfección unos objetivos y contenidos, sino 
que, como cualquier ser racional, en este caso dedicado a la docencia, interpreta, se deja llevar por su experiencia y pone en marcha, total o parcialmente, un método en una clase concreta con unos alumnos determinados en un tiempo y un lugar dados, es también, al fin y al cabo, un ser humano.

I am a Jew. Hath not a Jew eyes? hath not a Jew hands, organs, dimensions, senses, affections, passions? fed with the same food, hurt with the same weapons, subject to the same diseases, heal'd by the same means,(...) If you prick us, do not bleed? if you tickle us, do we not laugh? if you poison us, do we not die? ( Shakespeare, "the merchant of Venice":90)

Quizás no sea exactamente racismo lo que existe hacia los profesores de la L2, sino una tradición que se basa en un respeto mal entendido, que nos da libertad pero que nos aisla de las experiencias de los demás y hace impensable pedir consejo o ayuda si se quiere conservar una buena reputación. Nuestra experiencia y la de nuestros compañeros, aunque todos sabemos lo útil que es, podría serlo mucho más.

Y es a partir de la experiencia de donde nace nuestro interés por la investigación educativa como único método capaz de mejorar profundamente la enseñanza de idiomas.

En un pequeño pueblo, se alza un bonito y limpio colegio que hace las delicias de los alumnos en Prácticas. Uno de sus profesores, que imparte Idioma, colabora con nosotros, no sólo como un tutor de prácticas, sino que además permite que grabemos sus clases en vídeo para poder así mostrar a los alumnos el contexto en el que desarrollarán sus intervenciones. Además nos grabó su propia programación en audio como ilustración a la grabación en vídeo de la clase impartida.

Al comparar las grabaciones de vídeo y audio pudimos comprobar, en ésta última, que la descripción de su metodología, objetivos y desarrollo del proceso de enseñanza aprendizaje difería de lo que estaba grabado en vídeo. Los datos objetivamente observables y el tiempo dedicado a las diferentes actividades, el tipo de interacción que tenía lugar en el aula y la predominancia de las destrezas orales o escritas reflejados en el audio no se correspondían con lo que todos podíamos ver en la grabación de vídeo. También en aquellos aspectos que requerían una mayor interiorización, como la integración del aprendizaje significativo a la metodología, en el estudio, análisis e utilización de los conocimientos previos del alumno, en la evaluación o tipo de enfoque lingüístico, se veía dicha diferencia. Esta anécdota, que analizaremos más detenidamente, nos ha llevado a la reflexión y justifica, en cierta medida, nuestro estudio.

Una de las sesiones consistía en el análisis de una evaluación, que, como todos los profesores sabemos y los de Idioma con más razón, constituye el pozo en el que los objetivos más generosos, los contenidos más actualizados y las actividades más motivadoras se sumergen por la falta de tiempo, la carencia de objetividad y el exceso de trabajo. La evaluación se convierte en unas "pruebas objetivas" que no evalúan esos objetivos, contenidos y actividades, sino estructuras gramaticales, adquisición de vocabulario y a lo sumo, expresión escrita.

$\mathrm{Al}$ establecer un paralelismo entre los objetivos y contenidos de ciclo, elaborados por dicho profesor y sus propios objetivos del control, pudimos comprobar ,para nuestra sorpresa, que existía una gran diferencia. En los objetivos generales se buscaba: 
- La comprensión de la información global y específica de textos orales y escritos en situaciones habituales.

- La producción de textos escritos dirigidos a distintos interlocutores valorando el papel del texto escrito en la comunicación.

- La lectura de forma comprensiva de textos cortos relacionados con diversos contenidos del ciclo.

- La comprensión y utilización de las convenciones lingüísticas utilizadas por los franceses en situaciones habituales

- La utilización de experiencias previas de aprendizaje para desarrollar estrategias que les ayuden a la comunicación.

En la evaluación se intentaba comprobar:

- Si el alumno comprende de forma global textos orales y escritos relativos a objetivos y situaciones conocidas.

- Si el alumno escribe de forma sencilla textos cortos respetando los códigos de la L2.

- Si memoriza y reproduce textos orales respetando las variables de entonación $y$ ritmo.

- Si utiliza convenciones lingüísticas propias de la L2 en situaciones sociales.

- Si produce textos escritos secuenciados a partir de diagramas, dibujos, etc.

En las actividades de evaluación escrita, por el contrario, se le pedía a los alumnos que:

- Realizaran las transformaciones gramaticales del Presente al Pasado Compuesto y del Presente al Futuro y se sustituyera el Complemento Directo y el Complemento Indirecto por Pronombres Personales en las frases propuestas.

- Realizaran un dictado de un texto ya conocido por los alumnos y la utilización de frases sobre cuestiones cotidianas.

- Realizaran una traducción.

Los objetivos que el profesor buscaba con estas actividades de evaluación eran:

- Conocer el nivel de adquisición de los alumnos, buscando el por qué de su error.

- Reforzar el conocimiento de estructuras gramaticales que podían no haber sido comprendidas o asimiladas.

- Dar a conocer a los alumnos los criterios empleados para la evaluación.

- Despertar la capacidad crítica discutiendo los errores cometidos y valorar el trabajo bien hecho para así motivar al alumno.

Sin entrar a enjuiciar la metodología empleada en este caso, que no es en absoluto nuestra intención, nos limitaremos a establecer un estudio comparativo entre los objetivos de ciclo y los de evaluación. Podemos indicar, en primer lugar, que los textos orales y escritos de intención claramente comunicativa que aparecían como material lingüístico de 
los primeros, se habían convertido en frases descontextualizadas cuyo propósito era el permitir una transformación gramatical. Los interlocutores y la situación de comunicación no existían ni en un plano oral ni escrito, puesto que las frases o el dictado no estaban dirigidas ni a los alumnos, ni por los alumnos. Simplemente se trataba de una evaluación de otros objetivos que los anunciados previamente.

Analizando, con posterioridad, los objetivos del control enunciados por el profesor y la corrección de éste, pudimos comprobar que el nivel interactivo de los alumnos tampoco era respetado, ya que la corrección se había basado en preguntas cerradas e individuales, interrumpiendo constantemente el profesor al alumno cuando éste proponía o intentaba proponer una versión diferente de la "correcta".

Por poco que se conozca la enseñanza de Idiomas en situación real, estamos seguros de que esta situación no es, ni mucho menos, extraordinaria; y más que juzgada, debe ser comprendida. Hemos leído y también hemos enseñado a los futuros profesores de Idioma de qué manera la metodología audiovisual basada en el estructuralismo ha sido repudiada y sustituida por el enfoque funcional-comunicativo. Todo lo que "olía a antiguo" ha ido desapareciendo de las publicaciones, de los proyectos, de los objetivos y de los contenidos de las programaciones del profesorado y se ha sustituido por el enfoque comunicativo que ilustra los Proyectos de Centro y de Ciclo. Sin embargo, lo que ocurre en el aula es diferente. Los ejercicios gramaticales de sustitución, los dictados lingüísticos o las traducciones siguen dominado el panorama de la clase real y, en este caso concreto, de la evaluación sumativa. No queremos entrar aquí a valorar si estas prácticas deben desaparecer o no, ni si es posible su coexistencia con el enfoque de las orientaciones del D.C.B. Lo que sí queremos defender, y esta experiencia creo que lo demuestra, es que no se reflexiona lo suficiente sobre lo que se hace en el aula, ya que no se ha hecho el hincapié debido en lo valioso de la propia observación, que suministraría una información fundamental al docente. Podemos decir que no se han integrado las nuevas aportaciones teóricas académicas y oficiales con la realidad y práctica diaria del aula. Quizás el enlace podría estar en estas nuevas propuestas de investigación educativa que permitirán revisar lo que realmente ocurre en situaciones de interacción educativa para posteriormente reflexionar sobre ellas.

Volviendo al principal protagonista de nuestro artículo, el profesor de Idioma Extranjero, nos gustaría resaltar la consideración que se le presta en las Orientaciones Curriculares del Área de Lenguas extranjeras. En las Orientaciones específicas es el único lugar en el que se cree fundamental el trabajo en equipo de los maestros que imparten el área para realizar proyectos curriculares, compartir experiencias, confeccionar $e$ intercambiar actividades y, en una palabra, conseguir una línea coherente dentro de la misma Área. Aunque en principio no se habla de dificultades o problemas que pueden surgir en el aula, sino de alcanzar una línea coherente, el profesor tiene que ser un profesional que debe transmitir la lengua y ayudar a los alumnos a desarrollar una capacidad natural para comunicarse con otras gentes, (...) crear las condiciones adecuadas para que se produzca el aprendizaje en un ambiente relajado en el que los alumnos deben participar en la toma de decisiones sobre su aprendizaje así como ayudarles a que sean responsables de su aprendizaje, analicen sus problemas con la ayuda 
de sus compañeros y la suya propia Su tarea fundamental no es estar constantemente al frente de la clase, explicando o dirigiendo la actividad, sino preparando y organizando el trabajo oral y escrito; además, mientras los alumnos trabajan, el profesor les ayudará a resolver sus dificultades. El profesor deberá así mismo acostumbrar a los alumnos a tener experiencias satisfactorias al emplear la L2, pues esto los desinhibirá y les ayudará a usarla con más fluidez. Por último, el alumno debe servir de contraste a la actividad del profesor. Si da su opinion sobre las actividades realizadas, su propio progreso y la marcha de la clase en general, el profesor podrá ir ajustando más su labor docente a las necesidades de clase.

Estas pautas extraídas del Curriculo Oficial para el Área de Primaria plantean un nivel de exigencia para el que, tal vez, no esté preparado el profesor científica y didácticamente. Se trata de obligaciones planteadas como punto de partida, no como orientaciones en el desarrollo de una labor y no se valora, en absoluto, las individualidades de los profesionales en ejercicio que cuentan con una experiencia individual que en la mayoría de los casos ha enfocado la enseñanza de una L2 de una forma autodidacta.

Como aproximación al conocimiento del profesorado de L2 y su acción educativa, queremos aportar los resultados parciales de dos encuestas realizadas al profesorado de L2 en ejercicio en Córdoba y su provincia.

En primer lugar hemos seleccionado los datos cuantitativos de los apartados 4.4.Profesorado y 4.5.3.- Procedimientos de la encuesta sobre La enseñanza del Francés en la E.G.B., realizada en la Universidad de Córdoba por la Unidad Docente de Lengua Francesa en el año 1992 .

La entonces Escuela Universitaria de Formación del Profesorado de E.G.B. de Córdoba estaba elaborando sus nuevos planes de estudio y en concreto la Unidad docente de Lenguas extranjeras se consagraba a orientar la formación inicial del Maestro especialista en L2 según las directrices de la L.O.G.S.E. Un grupo de profesores de Francés y alumnos internos del Departamento de Filología Francesa e Inglesa y sus Didácticas del que formamos parte elaboró una encuesta para conocer el estado de la enseñanza del Francés en los Centro de E.G.B. de Córdoba y su Provincia.

El apartado 4.4. constaba del siguiente ítem:

- ¿El encuestado es profesor especialista en el idioma? 1-(sí), 2 -(no)

El apartado 4.5.3. constaba de los siguientes items:

\subsection{3. - Procedimientos}

4.5.3. 1. - Organización y desarrollo de las actividades

A - Asigne el porcentaje aproximado del tiempo total ocupado por cada tipo de las actividades siguientes:

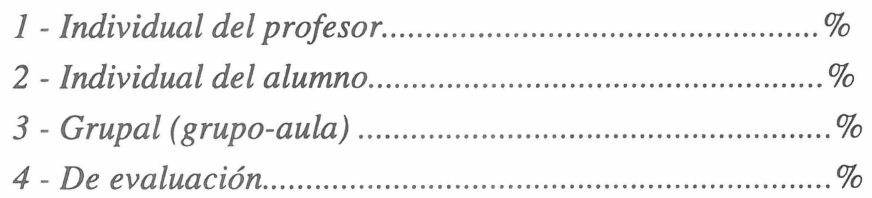


B - En las actividades antes reseñadas, ¿en qué proporción están las actividades orales y escritas?

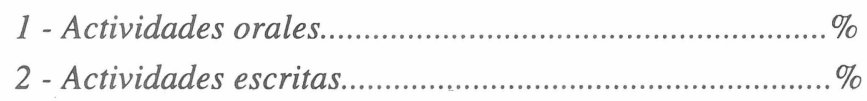

C - Indique, en porcentaje aproximado:

1 -Actividades impuestas por el profesor............................\%

2 -Actividades sugeridas por el profesor............................. \%

3 -Actividades elegidas libremente por el alumno...............\%

El objetivo de estos items era el siguiente:

En relación con el profesorado de L2 buscábamos establecer una relación entre la formación del profesor y la elección de su metodología, considerando para ello, la tipología de las actividades desarrolladas en el aula.

En cuanto a asignar un porcentaje de tiempo a cada una de las actividades, tomamos como variables la intervención del profesor o de los alumnos y las actividades de enseñanza-aprendizaje frente a las de evaluación. Nos interesaba saber si el profesor era consciente de su participación relativa y la distribución entre aprendizaje y evaluación.

Con el ítem B buscábamos también recabar información sobre la proporción entre las actividades correspondientes a destrezas orales y escritas.

Mediante la clasificación de las actividades dependiendo de la planificación individual o conjunta entre el profesor y los alumnos, intentábamos conocer el grado de intervención del alumnado y así comprobar el nivel de implantación de la metodología comunicativa y de las directrices del D.C.B.

El estudio de las respuestas nos permitè extraer las siguientes conclusiones:

La respuesta al ítem 4.4. nos daba la proporción siguiente:20 profesores especialistas y 4 no especialistas, sin que ello significara una modificación significativa en las respuestas al resto de los items. Los profesores no especialistas comparten con el resto la proporción media en la metodología y la tipología de las actividades.

En relación al ítem 4.5.3.1.A, relativo a la proporción entre las actividades (Fig. 1), nos sorprende, en todas las respuestas, la escasa intervención individual del profesor en el aula, que en el caso de mayor participación es del $33 \%$. Del mismo modo, la proporción de las actividades realizadas por los alumnos es muy elevada, lo que nos indicaría una metodología interactiva y un enfoque comunicativo de gran participación. Aparentemente esta tendencia parece verificarse mediante las proporciones relativas al uso de la Lengua oral o la Lengua escrita y al alto porcentaje de las actividades elegidas libremente por el alumno. 


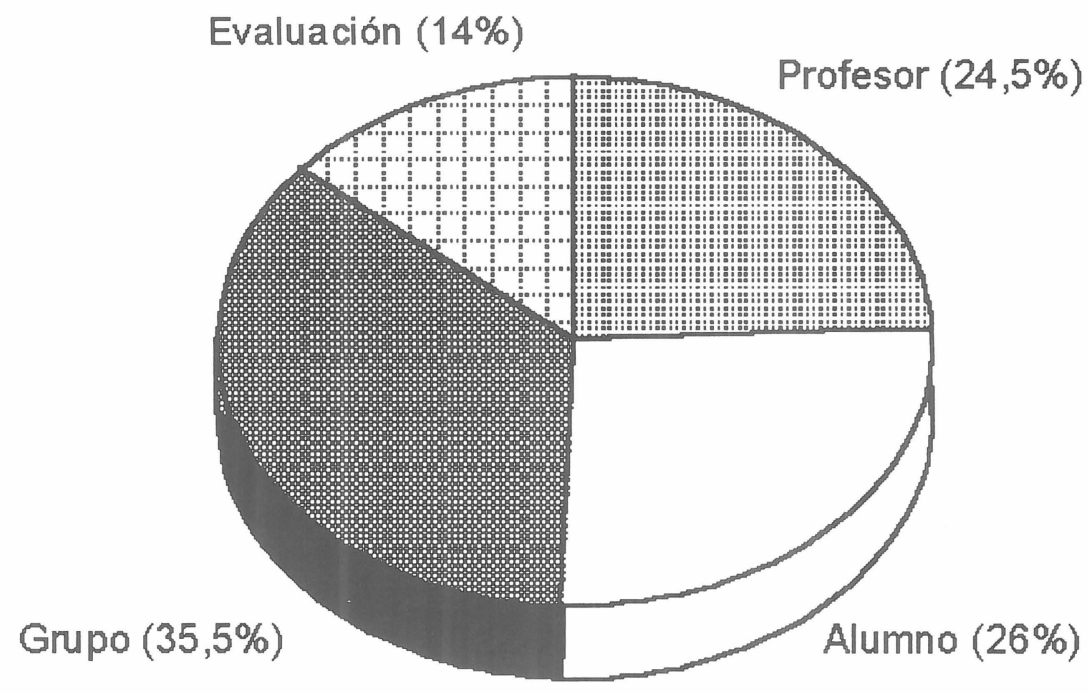

Figura 1. Porcentaje de tiempo dedicado a cada tipo de actividad.

Las actividades de aprendizaje (profesor+alumno+grupo) representan el $86 \%$

Nuestra sorpresa nos lleva a plantearnos la realidad de los datos, ya que las respuestas se alejan demasiado de la realidad.

Research in first language classrooms has established that teachers tend to do most of the talking (about $60 \%$ of the moves), mostly as soliciting and reacting moves. (...) In L2 classrooms, the research tends to support the conclusion from $L 1$ research. Teachers dominate classroom speech. (Chaudron.1988:50-51)

Una respuesta tan contundente como la recibida nos conduce a una profunda reflexión sobre la veracidad de las respuestas y a una hipótesis. Los profesores encuestados respondieron de acuerdo con lo que ellos pensaban que era "lo correcto" sin haber realizado el menor cómputo de la participación real ni de la tipología de las actividades, con lo que se hubiera puesto de manifiesto una proporción totalmente diferente.

En cuanto a la proporción entre destrezas orales y escritas, la mayoría oscila alrededor de una paridad aunque la desviación existente indica una prioridad de la Lengua oral sobre la Lengua escrita. Estos datos son, a nuestro parecer, susceptibles de duda ya que a lo largo de quince años dedicados a la tutoración de Centros de enseñanza y, en concreto, al seguimiento de las prácticas de Francés en Córdoba y su provincia, el enfoque de la enseñanza de esta lengua ha seguido la planificación de los libros de texto, de enfoque no comunicativo, en su gran mayoría, concediéndole una mayor importancia a las destrezas escritas. 


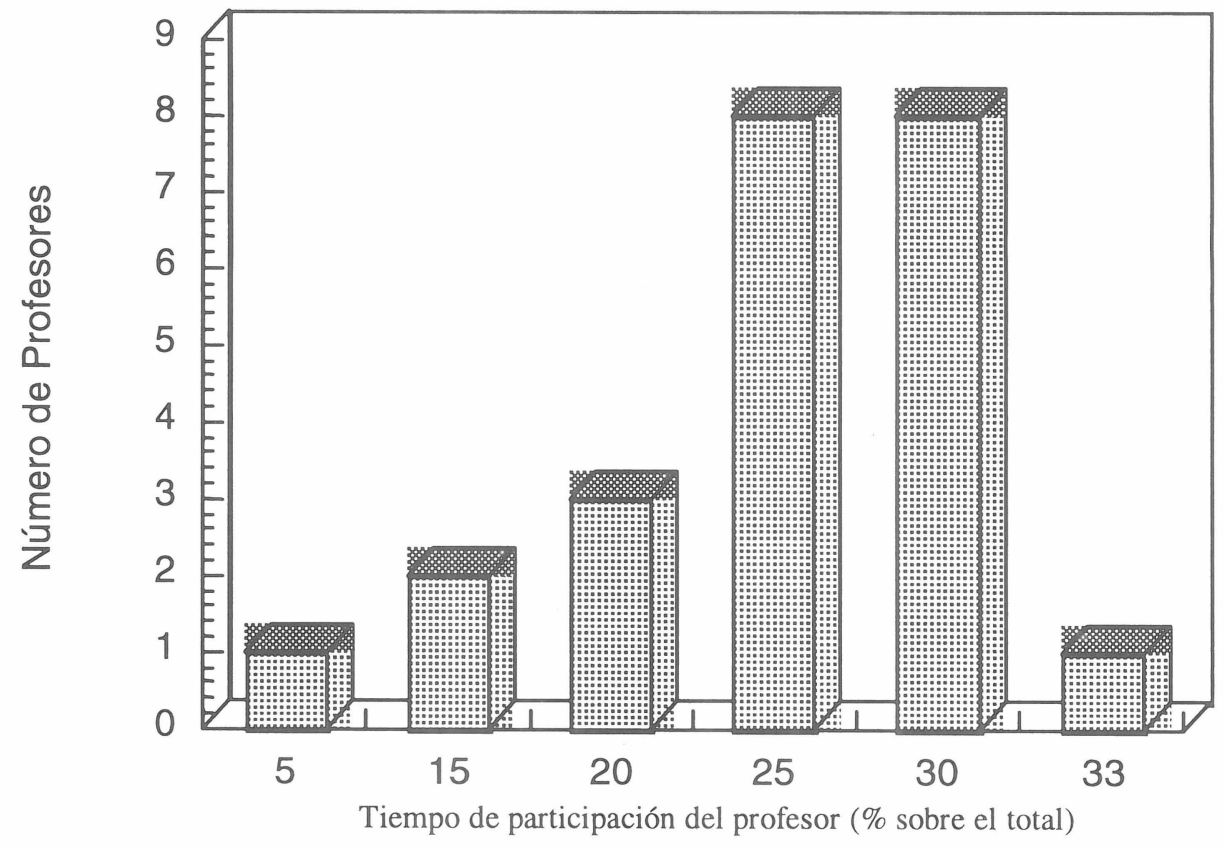

Figura 2. Índice de participación del profesor en las actividades del aula.

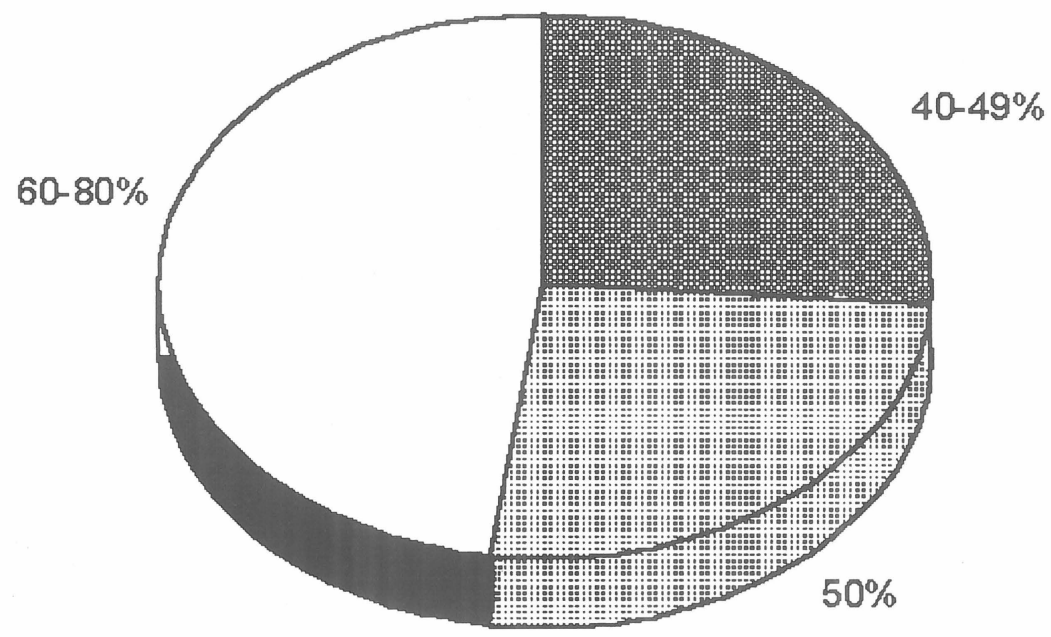

Figura 3. Proporción de actividades orales.

Respuestas correspondientes al item 4.5.3.1.B. 
Por último, aún nos sorprende más la proporción concedida en el ítem 4.5.3.1.C. a las actividades elegidas libremente por el alumno, puesto que el simple hecho de existir alguna indica una metodología muy avanzada en la enseñanza de los idiomas, como el trabajo por tareas o la simulación global.

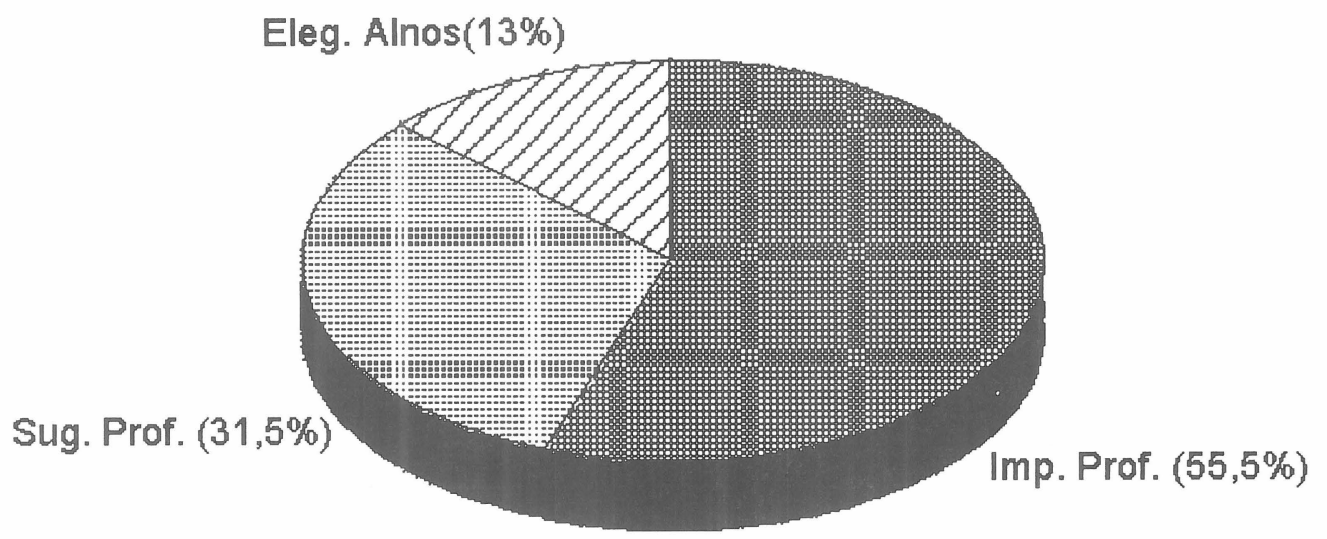

Figura 4. Planificación de actividades: profesor/alumno. Respuestas correspondientes al item 4.5.3.1.C.

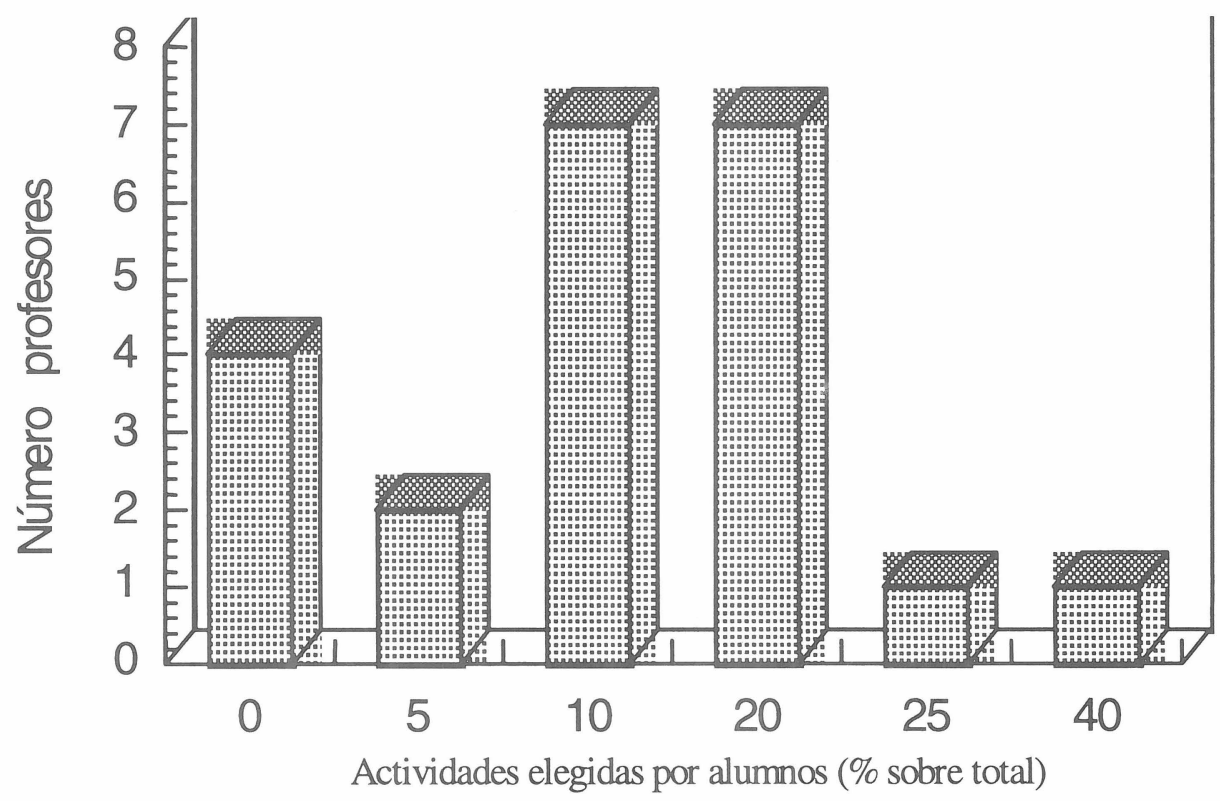

Figura 5. Proporción de actividades elegidas por los alumnos. 
Así pues, trabajar por tareas implica adoptar la tarea como unidad de diseño de la actividad didáctica. Es decir, el maestro al hacer la programación no parte de los contenidos lingüísticos (nociones, funciones, estructuras) y basándose en ellas diseña actividades, sino al contrario: programa tareas finales y a partir de éstas aborda las tareas intermedias, los objetivos didácticos, los contenidos, la metodología y la evaluación de la propia tarea. (...)

Es conveniente que los alumnos elijan los temas que más les gusten, para ello el maestro les puede dar una lista y pedirles que voten los que les resulten más atractivos. (Lenguas extranjeras Primaria. M.E.C. 1992: 116)

El trabajo por proyectos parte de los mismos principios teóricos y utiliza una metodología similar a la del trabajo por tareas. (...) Cada proyecto es el resultado de un trabajo cooperativo que empieza con la decisión del tema, búsqueda de materiales, trabajo individual y en equipo, maquetación y forma coherente y la más creativa posible de desarrollo y presentación. (ibid: 116)

La simulación global podría definirse como un caso particular de trabajo por proyectos. (...) Presenta numerosas ventajas, pues en ella se alternan la búsqueda de ideas, la creatividad colectiva, los juegos de rol, la improvisación y las producciones escritas. La clase de lengua se convierte, pues, en un gran juego en el que los actores principales son los alumnos. Además le permite la toma de decisiones y la implicación correspondiente en las iniciativas que se lleven a cabo. (ibid: 117).

La segunda de las encuestas realizadas debe enmarcarse en nuestra corta experiencia como responsables de la materia correspondiente a la lincenciatura de Psicopedagogía: Intervención didáctica en el Area de Idiomas. En el curso 94-95, uno de los trabajos sugeridos al alumnado fue la elaboración de encuestas sobre la enseñanza de L2 en los niveles de Primaria y Secundaria. Los alumnos siguieron las pautas de método cuantitativo de investigación, consistente en cuestionarios dirigidos a profesores y alumnos, utilizando para ello preguntas abiertas y cerradas. Los temas tratados en las encuestas realizadas a profesores se podrían agrupar de la siguiente manera:

A: Datos de situación

B: Metodología

- Líneas generales:

1- Tipos de contenidos

- Procedimentales

- Actitudinales

- Conceptuales

2- Tipos de destrezas

- Lengua oral

- Lengua escrita

3- Porcentaje de utilización de la L1 y L2 en el aula.

4- Metodología propiamente dicha

- Criterios

- Estrategias

- Posibles mejoras

5- Agrupamientos

- Trabajo en grupo

-Tipos de agrupamiento 


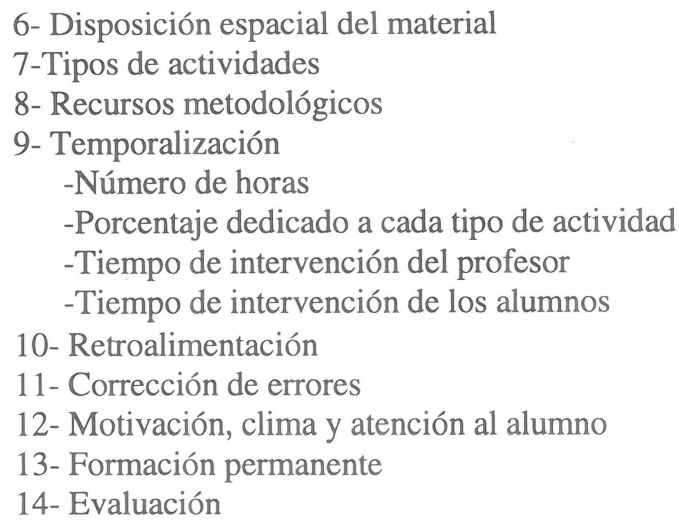

(Debido a lo extenso y variado de los modelos de cuestionarios nos limitamos a reseñar los comentarios más significativos de los autores de las encuestas sobre el resultado de las mismas):

En relación con los datos de situación:

- La media general de la muestra analizada oscila entre los 30 y 40 años. No existe ningún profesor nativo. Entre los de mayor edad, la titulación para impartir el idioma ha sido adquirida, en la mayor parte de los casos, a través de un cursillo de especialización.

La mayoría del profesorado encuestado, profesorado de Primaria, es Diplomado en Filología Inglesa, y considera los cursillos adecuados para "ponerse al día", cosa que en el idioma es una necesidad. Deducimos que en el área de Idiomas, se debería incluir este tipo de cursos de formación dentro del horario lectivo, para poder seguir una línea unificada y evitar la repetición de los contenidos que algunos profesores señalan.

El profesorado de Secundaria está formado por licenciados en Inglés la mayoría, y el profesorado encuestado licenciado en Francés, se ha iniciado en otro idioma (uno de ellos en Inglés y otro en Italiano) lo cual muestra la incertidumbre de estos últimos. En lo que se refiere a los cursillos de formación y perfeccionamiento, el profesorado de Secundaria es más exigente que el de Primaria y aunque los considera frecuentes, no siempre los ve adecuados o de interés.

Podemos observar que se le ha dado una gran importancia, tanto por parte de los alumnos que han elaborado la encuesta, como de los profesores que la han respondido, al hecho de tener una adecuada preparación para impartir el Idioma y, lo que es fundamental desde nuestro punto de vista, al hecho de que el profesor de Idiomas debe estar en constante formación, ya que se considera insuficiente la obtenida con los cursillos de formación y perfeccionamiento a los que tienen acceso. El hábito de investigar en el aula podría paliar algunas de las deficiencias y animaría al profesorado a revisar su docencia.

En cuanto a los contenidos, las conclusiones extraídas por los alumnos señalan que casi todos los profesores priorizan los contenidos procedimentales y actitudinales. Asimismo consideran que existen diferencias a la hora de tratar la lengua oral o escrita.

Desglosados los datos de los once cuestionarios obtenemos los siguientes resultados: 
De una forma similar a la primera encuesta, la respuesta correspondiente al ítem $\mathrm{n}^{\circ} 2$ establece, de una forma paritaria, la proporción entre las destrezas orales y escritas, lo cual refleja, más que un esfuerzo por parte del profesorado en hacer equivalentes ambas destrezas, un desinterés por la pregunta, que se ha respondido grosso modo. En los items 7 y 9 , al tener que pormenorizar la tipología de las actividades, y el tiempo dedicado a cada una de ellas, se demostraba dicha ausencia de rigor.

En cuanto al ítem 9, donde había que establecer la proporción de las intervenciones entre el profesor y el alumno, creemos que son inexactas.

Respuesta A: $66 \%$ profesor / $33 \%$ alumnos Respuesta B: $65 \%$ profesor $/ 35 \%$ alumnos Respuesta C: $25 \%$ profesor $/ 75 \%$ alumnos

Respuesta D: $25 \%$ profesor $/ 75 \%$ alumnos Respuesta E: $25 \%$ profesor/ $75 \%$ alumnos Respuesta F: $25 \%$ profesor $/ 75 \%$ alumnos Respuesta G: $25 \%$ profesor $/ 75 \%$ alumnos Respuesta $\mathrm{H}: 50 \%$ profesor / $50 \%$ alumnos Respuesta I : $50 \%$ profesor / $50 \%$ alumnos Respuesta J: $33 \%$ profesor / 66\% alumnos Respuesta K: $50 \%$ profesor / $50 \%$ alumnos

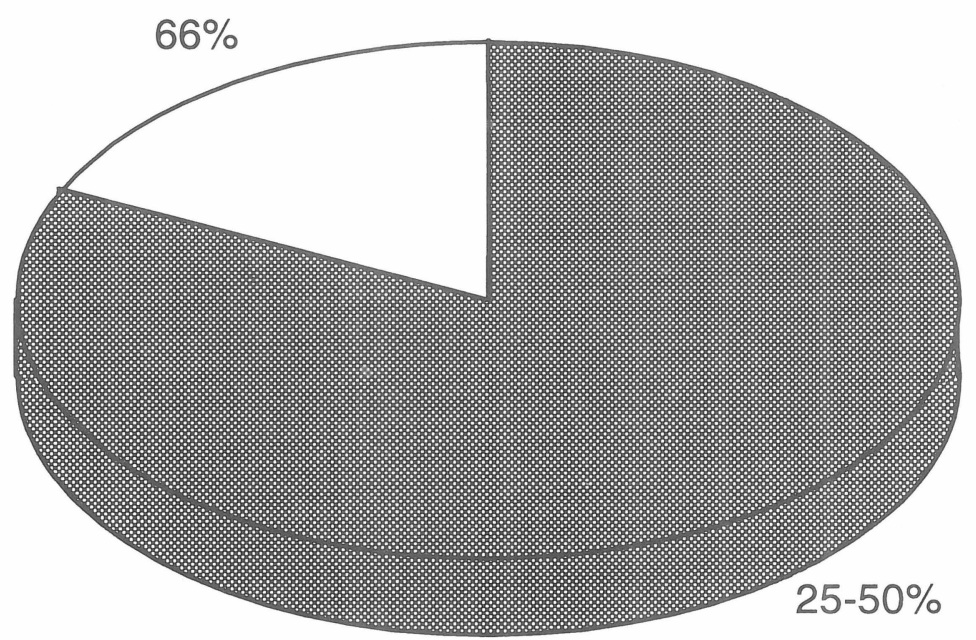

Figura 6. Intervención proporcional profesor/alumno.

Para cualquier persona que se haya detenido a estudiar este aspecto en el aula, es evidente que todo porcentaje que no supere un $75 \%$ de participación, por parte del profesor, es altamente improbable; y porcentajes por debajo de un 50\%, imposibles en Primaria. A 
medida que el conocimiento de los alumnos mejora, también aumenta el porcentaje de participación de estos. En Secundaria, en una enseñanza de enfoque comunicativo, en la que los alumnos estén acostumbrados a trabajar en grupos utilizando la L2 en su interacción, sí se puede alcanzar un porcentaje de participación del alumnado bastante elevado.

Hemos podido comprobar, a este respecto, que, cuando se empieza a trabajar con técnicas de observación, la primera impresión de los alumnos, al ver la grabación en vídeo u oír la cassette es expresar su sorpresa por lo mucho que habla el profesor, incluso en clases poco tradicionales.

Desde el punto de vista del profesor, es muy difícil darse cuenta cuando se está impartiendo una clase de idioma de lo "mucho" que habla él y de lo "poco" que lo hacen los alumnos. Las consecuencias de este desconocimiento influyen a la hora de seguir las Directrices Generales de tipo metodológico del D.C.B., basadas en la interacción dentro del aula, pues la interacción evidentemente implica la participación por parte del alumnado.

Not surprisingly, classroom observation has revealed that teachers tend to do most of the talking. In content classrooms (e.g. science, mathematics classrooms) it has been found that teachers tend to talk for about two-thirds of the available class time, leaving just a third for learners. In some language classrooms it has been shown that teachers talk for up to 89 per cent of the available time.

Whether this is a good thing or not will depend on what one believes about the role of language input in acquisition. If one believes that learners learn best by actually practising in the target language, one will probably try to structure classroom activities so that amount of learner talk is increased at the expense of teacher talk. If, on the other hand, one believes that teacher talk is a valuable of comprehensible input, one will be much less worried by teacher dominance.

Teachers who undertake to record and analyse their classroom interactions are generally disconcerted at the quality and quantity of their talk. In Workshops with teachers, I have found that particular areas of concern are the amount of time they talk and the nature of the instructions and explanations they provide. (Nunan 1989:26)

Debemos subrayar también que la mayoría de los profesores encuestados había señalado la dificultad de llevar a cabo una metodología comunicativa debido al elevado número de alumnos, lo que está en franca contradicción con la gran participación, supuestamente, de los alumnos y los objetivos a los que se les da una mayor prioridad, procedimentales, y actitudinales. Tal y como los autores de esta encuesta señalan, Por las contradicciones que hemos visto, observamos que hay una mayor preocupación por los aspectos mofosintácticos y gramaticales, que por los de carácter práctico

Podemos añadir algunos datos extraídos de la encuesta realizada al profesor A que indican que su metodología es activa, práctica y participativa así como estructural utilizando, sin embargo, la L1 como base para la enseñanza y el cuaderno de trabajo para controlar las actividades diarias y educar en la buena organización y en la tarea diaria. El tipo de comunicación empleado se centra en diálogos con el profesor, vocabulario, cartas, peticiones y, dentro de las actividades, la lectura, el afianzamiento, preguntas sobre un texto, ejercicios estructurales, conceptos de gramática. En la evaluación indica que realiza 
el control de forma detenida, valorando la importancia de los errores a la hora de puntuar, escribiendo las respuestas correctas debajo, poniendo notas orientadoras que puedan servir de motivación y de llamada al alumno. Este método ecléctico presenta, a nuestro modo de ver, contradicciones a primera vista: utilización de lengua oral en el desarrollo de las clases y controles en lengua escrita, metodología activa y participativa en enseñanza individualizada, ejercicios estructurales y dependencia del libro de texto. Todo ello, de todas formas, no son más que conclusiones hipotéticas, pues habría que ver qué ocurre realmente en el aula. Ello no hace sino demostrar nuestra consideración a este tipo de encuesta como método no suficiente para obtener datos objetivos y, por tanto, la necesidad de la investigación en el aula.

Algunos de los cuestionarios realizados en Secundaria apoyaban un enfoque funcional dándole un mayor papel al lenguaje escrito, orientación que quedaba reflejada en el tipo de evaluación. A pesar de que esta orientación está perfectamente justificada dado el desarrollo cognitivo de los alumnos y sus perspectivas de uso del idioma extranjero, no existía un convencimiento, por parte del profesor, de que éste era el mejor enfoque escogido por él. Detectamos, pues, una necesidad de justificación del enfoque empleado, ya que el lenguaje oral parece que actualmente "está mejor visto" y el escrito representa, sin embargo, la enseñanza tradicional o responde a una insuficiente competencia lingüística.

Creemos interesante pormenorizar algunas de las respuestas a los items pertenecientes a los cuestionarios planteados a los profesores de Secundaria.

7- Tipos de actividades

7.1. - ¿ A qué actividades das prioridad?

14- Evaluación

14.1.- ¿Qué resultados obtienes? ¿Los valorarías como positivos?

El profesor que respondió al cuestionario $n^{\circ} 6$ indica: 7.1. Actividades de tipo escrito (dado el elevado número de alumnos) Como respuesta a la pregunta sobre evaluación 14.1. Negativos, ya que el excesivo número de alumnos impide al profesor atenderlos adecuadamente

Las respuestas en la encuesta $n^{\circ} 7$ como contestación a las mismas preguntas eran las siguientes: 7.1 Actividades de tipo escrito por las circunstancias de un excesivo número de alumnos en clase y la incapacidad de evaluarlos oralmente, 14.1. Negativos, debido al excesivo número de alumnos y a la poca motivación de algunos.

La respuesta a la encuesta $\mathrm{n}^{\circ} 8$ insistía en el mismo aspecto indicando: 7.1. escrito, por no poder corregir la pronunciación de forma personal e individualizada. En cuanto a la evaluación, señala: 14.1. Le doy mayor importancia a la asimilación de estructuras y la corrección gramatical ; ya que la pronunciación por lo anteriormente dicho, no puede priorizarse.

Estas respuestas nos llevan a reflexionar sobre un nuevo aspecto dentro de las opiniones del profesorado sobre lo que hay que hacer y lo que se hace en el aula. La afirmación conocida acerca de un alumno que "lleva muchos años estudiando el idioma pero que no puede expresarse oralmente en ese idioma" supone una crítica al sistema de enseñanza de 
L2 que parece aterrorizar al profesorado, el cual adopta una actitud de auto-defensa, como hemos podido constatar en las anteriores afirmaciones. Esto no hace sino alargar la distancia que existe entre el conocimiento de lo que el profesor de L2 hace en el aula y sus objetivos, ya que éstos están totalmente condicionados por un factor negativo, insuperable al parecer, el excesivo número de alumnos. Ese número de alumnos, sin embargo, es muy similar al que imparten docencia los profesores de Primaria, los cuales, aunque señalan este factor como negativo, no estiman que ello impida una enseñanza basada en destrezas orales.

Por último, subrayamos una afirmación casi general por parte del profesorado encuestado: la necesidad de coordinación entre el profesorado que imparte idioma. Afirmación que contrasta con los datos: sólo el $50 \%$ de los profesores afirma que existe esa coordinación.

La valoración e interpretación de los datos recogidos de las encuestas, así como el análisis más detenido de algunos ejemplos, muestran el desconocimiento general por parte del profesor, de cómo se desarrolla la enseñanza y aprendizaje de L2 en su aula. Esto nos lleva a concluir que la utilización de cuestionarios en los que no está implicada la reflexión del profesor no es válida como comprobación de su actuación en el aula. Por todo ello creemos que podemos finalizar nuestro trabajo sugiriendo dos líneas de investigación educativa específicas para el profesor de L2:

La primera de ellas partiría del estudio del profesor en tanto que organizador del aula, elector de una metodología y actor en la interacción profesor-alumno. Organización, elección y acción que constituyen el desarrollo material y patente de un conjunto de conocimientos y teorías implícitas que se han ido configurando a lo largo de su experiencia como docente. El procedimiento a seguir consiste en analizar y por lo tanto dar un primer paso para resolver la contradicción entre la filosofía o las ideas sobre educación, declaradas públicamente por el profesor, y el comportamiento de éste en el aula. De este modo el docente evitaría las contradicciones entre sus metas y objetivos y la acción propiamente dicha.

The research, as Shavelson and Stern point out, is based on two fundamental assumptions. The first of these is that teachers are rational beings whose work requires them to make decisions in complex environments. The second is that what teachers actually do in class is guided by their thoughts, judgments and decisions which in turn will be influenced by their training, personal characteristics, etc. The research reminds us that in order to understand classrooms we may sometimes need to go beyond the actual classroom situation itself. (Nunan. 1989: 20-21)

Esto nos conduce a sugerir que, también a nivel de la formación inicial del profesor, es necesario analizar los conceptos educativos, ya que ellos van a influir en la adquisición de los presupuestos teóricos de la enseñanza-aprendizaje de la L2 y condicionar las prácticas de enseñanza de su formación como docente. Todo ello tendrá una repercusión importante en los primeros años de ejecicio del profesor en los que se construyen las metodologías propias.

As we seek to move second language teaching away from the status of "magic" or "art" and toward the status of profession, an important goal will be public recognition of the existence of the field of second language teaching as such. Part of the professionalization of 
language teaching involves the codification of the knowledge of the field into explicits goals for teacher preparation and effective methods for achieving these goals. For continuing career growth and individualized development, primary goals are:

- a knowledge of theorical base of the field in language learning and classroom research;

- informed knowledge of self students;

- attitudes of flexibility and openness to change;

-decision-making and communication skills;

-the analytical skills necessary for assessing different teaching situations and the changing conditions in a classroom;

- awareness of alternative teaching approaches and the ability to put these into practice;

- the confidence and the skills to alter one's teaching approach as needed;

- practical experience with different teaching approaches.

Methods that involve the future teacher in practical experiences - both simulations and real- life teaching experiences - and in analysis of these, and which are grounded in educational experiences that include theory and research constitute a logical preparatory base for the lifelongs second language professional. Such methods, which apply the combined insights and skills of these practical and intellectual strands in a practicum component, are fundamental part of a coherent language-teacher education curriculum directed at the longterm development of its graduates. (Pennington. 1990:150-151)

La segunda línea de investigación que proponemos debe centrarse en la actuación del profesor de forma que éste sea consciente de ella y pueda compararla con su planificación. Esto le permitirá seleccionar aquellos aspectos que desee mejorar aplicando el método de investigación más adecuado para ello.

Como hemos intentado demostrar mediante nuestro análisis, no toda la investigación educativa suministra datos objetivos sobre la actuación del profesor; a esto podemos añadir el hecho de que muchos de los métodos sobre el aprendizaje del idioma no se centran en el contexto de aula propiamente dicho.

When one examines the literature on classroom observation and research one is struck by the relative paucity of research as Lemke's where the data were actually collected within genuine classrooms. (As I have said, by "genuine" classroms, I mean classrooms which were specifically constituted for the purposes of teaching and learning, not to provide a venue for research.) (...) The survey revealed that of the fifty studies, only fifteen were actually carried out in genuine language classrooms. A further seven collected some of their data in classrooms, and some of their data out of class. The great majority collected their data outside the classroom in laboratory, simulated, and naturalistic setting. Despite this, the researcher had no hesitation in claiming pedagogic relevance for their research. (Nunam.1992: 102-103)

Aunque tanto el método experimental como el etnográfico gozan de una amplia difusión en la investigación educativa, desde nuestra experiencia concreta creemos que sólo puede aplicarse en casos muy particulares. Además necesita de conocimientos o medios difíciles de alcanzar al no existir, en la mayoría de los casos, grupos de investigación experimentados.

En relación al estudio de casos, centrado en una única experiencia que puede llegar a extrapolarse, pensamos que afecta escasamente a la planificación-acción del profesor. 
Coincidimos, por tanto, con D.Nunan en señalar que una investigación particularizada debe elegir, en primer lugar, la técnica más apropiada de observación e investigación en el aula dentro del amplio espectro que va desde el experimento formal o los esquemas para la observación y análisis de la interacción, a la estimulación del recuerdo. Si el profesor desea observar y reflexionar sobre los propios pensamientos, podrá utilizar tanto técnicas introspectivas ( verbalización de pensamientos o procesos lógicos, diarios) como técnicas retrospectivas; caracterizadas las primeras por ser coetáneas y las segundas por mediar un espacio de tiempo entre la acción y su recuerdo.

Todo ello debe estar siempre en función del aspecto seleccionado por el profesor, individualmente o en conexión con el observador externo.

Existe una amplia muestra de técnicas de obtención de datos entre los que el investigador deberá hacer una selección: entrevistas, cuestionarios, o estudios de casos y por último, deberá realizar una evaluación de todo el proceso a fin de cumplir los requisitos necesarios para ser fiable y veraz.

Se puede optar por escoger un método completo de investigación (de carácter exclusivamente cuantitativo, cualitativo o mixto) que incluya una elección de cada uno de los apartados anteriormente citados, o construir mediante la libre elección, un método adecuado a la situación particular. Toda una amplia gama de posibilidades está abierta a quien desee mejorar o simplemente conocer, cómo realiza cotidianamente su trabajo. 


\section{BIBLIOGRAFÍA}

K.A.ACHENSON, Techniques in the Clinical Supervision of teachers. (New York 1992) Longman.

R.ALLWRIGHT, K.M.BAILEY, Focus on the language Classroom: An Introduction to Classroom Research for Language Teachers. (Cambridge 1991) C.U.P.

R.ALTMAN, The video connection. Integrating video into language teaching. (Boston 1988) Houghton Mifflin Company.

J.P.BASAILlE, D.FLAMENT, M.L.LIONS, J. PÉCHEUR, La Didactique au quotidien. Actes du colloque de Toulon. Sept. 1994. Le Français dans le monde, numéro spécial. (1995) E.D.I.C.E.F.

B.BEASLEY, L.RIORDAN, "The classroom teacher as researcher". English in Australia, 55 (1981) 56-63.

P.BERTOCHINI, E.CONSTANZO, Manuel d'autoformation. (Paris 1989) Formation Hachette.

P.BOGAARDS, Aptitude et affectivité dans l'apprentissage des langues étrangères. (Paris 1988) L.A.L. Crédif Hatier.

P.BOGAARDS, Le Vocabulaire dans l'apprentissage des langues étrangères. (Paris 1994) Crédif Hatier Didier.

S.BOLTON, Évaluation de la compétence communicative en Langue étrangère. (Paris 1987) L.A.L. Crédif Hatier.

H.BOYER, M.BUTZBACH, M.PENDANX, Nouvelle introduction à la didactique du français langue étrangère. (Paris 1990) CLÉ International.

T.BOWEN, J.MARKS, Inside teaching. (Oxford 1994) Heinemann.

R.BOWERS, C.BRUMFIT, Applied Linguistics and English language teaching. (Developments in E.L.T. Series) (Hong Kong 1994) E.L.T.

H.D.BROWN, Principles of Language Learning and Teaching. (New Jersey 1993) Prentice Hall Regents.

H.BROWN, H.DOUGLAS, Teaching by principles. (New Jersey 1994) Prentice Hall Regents

C.BRUNMFIT y R.MITCHELL, (eds) Research in the Language Classroom. (London 1990) Modern English Publications

D BYRNE, Techniques for Classroom Interaction. (London 1987) Longman.

C CANDLIN, "Hacia la enseñanza de lenguas basada en tareas". Comunicación Lenguaje y Educación. 7-8 (1990) 33-53

R.CARTER y R.McCARTHY, (eds.). Vocabulary and Language teaching. (London 1988) Longman. 
C.CHAUDRON, Second Language Classrooms: Research in Teaching and Learning. (Cambridge 1988) C.U.P.

R.COOPER, M.LAVERY, M.RINVOLUCRI, Video. (Oxford 1991) Oxford University Press.

A.DE LA GARANDERIE, Comprendre et imaginer. Les gestes mentaux et leur mise en oeuvre. (Paris 1987) Editions du Centurion.

G.DELAIRE, H.ORDONNEAU, Enseigner en équipe. Les guides du métier d'enseignant. (Paris 1989) Ed.Organisation.

L.DICKINSON, Learner Training for Language Learning. (Dublin 1992) Trinity College

J.C.DORTU, L'analyse transactionelle à l'école. Les guides du métier d'enseignant. (Paris 1992) Ed.Organisation.

P.DUNKEL, (ed) Computer-Assisted Language Learning and Testing: Research Issues and Practice. (New York 1991) Newbury House.

R.ELLIS, Instructed Second Language Adquisition: Learning in the Classroom. (Cambridge 1990) Basil Blackwell.

R.ELLIS, "Activities and procedures for teacher preparation" en J.RICHARDS y J.RICHARDS y D. NUNAN. (eds) Second Language Teacher Education. (New York 1990) C.U.P. 26-36.

G.ELLIS, B.SINCLAIR, Learning to Learn English. (Cambridge 1989) Cambridge University Press.

S.ESTAIRE, J. ZANON, Task-based Teaching (Oxford 1994) Heinemann.

D.FREEMAN, "Teacher Training Development and Decision-Making. A model of Teaching and Related Strategies for Second Language Teacher Education", en TESOL Quaterly. 23:2, (1989) 27-45.

D.FREEMAN, "Intervening in practice teaching" en J.RICHARDS y D. NUNAN. (eds) Second Language Teacher Education. (New York 1990) C.U.P. 103-117.

D.FREEMAN, "Collaboration: constructing shared understandings in a second language classroom" en D.NUNAN.(ed) Collaborative Language Learning and Teaching. (Cambridge1992) 56-80 C.U.P.

R.GADNER, Social Psychology and Second Language Learning. The Roles of Attitude and Motivation. (London 1985) Edward Arnold.

R.GALISSON, E.ROULET, Vers une didactique du Français. Langue Francaise 82. Mai (1989) Larousse.

D.GAONAC'H, Théories d'apprentissage et acquisition d'une langue étrangère. (Paris 1987) L.A.L Hatier Crédif

S.GASS, S.MADDEN, D.PRESTON, L.SELINKER. Variation in Second Language Acquisition: Discourse and Pragmatics. (Clevedon Philadelphia 1989) Multilingual Matters. L.T.D. 
J.G.GEBHARD, Interaction in a teaching practicum en J.RICHARDS y D. NUNAN. (eds) Second Language Teacher Education. (New York 1990) C.U.P. 118-131.

B.N.GRUNÏG, La fuite du sens. La construction du sens dans l'interlocution. (Paris 1985) L.A.L. Crédif Hatier.

S.HALliWELL, La enseñanza del Inglés en la Educación Primaria. (Madrid 1993) Longman.

J.B.HEATON, Classroom Testing. (New York 1990) Longman.

D.HOPKINS. Investigación en el aula. (Barcelona1989) P.P.U.

C.JAMES, P.GARRET.(eds.) Language Awareness in the Classroom. (Harlow 1991) Longman.

S.KELLERMAN, "Lip service. The contribution of the visual modality to speech perception and its relevance to the teaching and testing of foreign language listening comprehension". Applied Linguistics. 11/3 (1990) 272-280.

W.KLEIN, Second Language Adquisition.(Melburne 1986) C.U.P.

V.KOHONEN, "Experiential language learning: second language learning as cooperative learner education", en D.NUNAN.(ed) Collaborative Language Learning and Teaching. (Cambridge 1992) 14-39 C.U.P.

R LEBLANC, "Le curriculum multidimensionel: Une synthèse", en The Canadian Modern Language Review, 47:1 (1990) 32-43.

D.LARSEN-FREEMAN, M.H.LONG, An Introduction to Second Language Adquisition Research. (London 1991) Longman.

M.LEGUTKE, H.THOMAS, Process and Experience in the Language Classroom. (New York 1991).Logman.

J.LONERGAN, “A decade of development: educational technology and language learning" Language Teaching. 24 (1991) 1-10.

D.LUISSIER, Évaluer les apprentissages dans une approche communicative. (Paris 1992) Hachette.

M.E.C. Primaria. Área de Lenguas Extranjeras. (Madrid 1992)

M.E.C. Optativas. Segunda Lengua Extranjera (Barcelona 1992)

P.MEIRIEU, Apprendre. Oui...mais comment? (Paris 1991) E.S.F.

P.MEIRIEU. L'école mode d'emploi. (Paris 1991) E.S.F.

S.MOIRAND, Enseigner à communiquer en langue étrangère. (Paris 1982) Hachette.

J.MUÑOZ LICERAS, La adquisición de lenguas extranjeras. (Madrid 1991) Visor

J.NARCY, Apprendre une Langue étrangère. (Paris 1990) Ed.Organisation.

D.NUNAN, Collaborative language learning and teaching. (Glasgow 1992) C.U.P.

D.NUNAN, Research methods in language learning. (New York 1992) C.U.P 
D.NUNAN, Understanding language classrooms. (Cambridge 1989) C.U.P.

D.NUNAN, "Action research in the language classroom" en J.RICHARDS y D. NUNAN. (eds) Second Language Teacher Education. (New York 1990) C.U.P. 62-81.

I.S.P.NATION, "Beginning to learn foreign vocabulary: a review of the research". RELC Journal 13/1 (1982): 14-36.

I.S.P.NATION, Teaching and Learning Vocabulary. (New York 1990) Newbury House.

J.M.O'MALLEY, A.U.CHAMOT, Learning Strategies in Second Language Acquisition. (Cambridge 1990) C.U.P.

R.OXFORD, Language Learning Strategies. What every teacher should know. (Boston Massachussetts 1990) Heinle and Heinle.

J.PÉCHEUR y G.VIGNER, "Méthodes et Méthodologies" Le Français dans le monde. Numéro spécial. (1995) E.D.I.C.E.F.

P.PELPEL, Se former pour enseigner. (Paris 1986) Bordas.

M.C.PENNINGTON, "A professional development focus for language teaching practicum" en J.RICHARDS y D. NUNAN. (eds) Second Language Teacher Education. (New York 1990) C.U.P. 132-151

CH.PUREN, Histoire et formation en Didactique des langues étrangères: quelques réflexions prospectives en Etudes de Linguistique appliquée.75. Juillet-Septembre (1989) 109-113.

P.REA-DICKINS, K.GERMAINE, Evaluation. (Oxford 1992) Oxford University Press.

R.D.RICHARD, "Teacher observation in second language teacher education" en J.RICHARDS y D. NUNAN.(eds) Second Language Teacher Education. (New York 1990) C.U.P. 43-57.

J.RICHARDS, D. NUNAN.(eds) Second Language Teacher Education. (New York 1990) C.U.P.

J.RICHARDS, "The dilemma of teacher education in second language teaching" en J.RICHARDS y D. NUNAN.(eds) Second Language Teacher Education. (New York 1990) C.U.P.

R.RIBÉ, N.VIDAL, Projet Work. Step by Step. (Oxford 1993) Heinemann.

R.RIBÉ, La enseñanza de la Lengua Extranjera en la Educación Secundaria. (Madrid 1995) Alhambra Longman.

L.SCHIFFLER. Pour un enseignement intéractif des langues étrangères. (Paris1984) Crédif Hatier.

H.W.SELIKER, Second Language Research Methods (Oxford 1989) Oxford University Press.

P.SKEHAN, Individual Differences in Second-Language Learning. (London 1989) Edward Arnold. 
N.B. SHANON, M.L. BONNIE, "Collaborative language teaching: a co-investigation”. en D.NUNAN. (ed) Collaborative Language Learning and Teaching. (Cambridge1992) 120-140. C.U.P.

H.H.STERN, Fundamental Concepts of language teaching (Oxford 1994) Oxford University Press.

C.TAGLIANTE, L'évaluation. Techniques de classe. (Paris 1991) CLÉ.

H.TROCME, "J'apprends donc je suis". (Paris 1987) Ed. Organisation.

G.VIGNER. Didactique fonctionnelle du français. (Paris 1980) Hachette.

M.WALLACE. Training foreign language teachers. (Glasgow 1991) C.U.P. 
